\title{
Clostridium difficile Infection at Diagnosis and during the Disease Course of Pediatric Inflammatory Bowel Disease
}

\author{
Do Hyun Kim*, Jin Min Cho*, and Hye Ran Yang*, $†$ \\ ${ }^{*}$ Department of Pediatrics, Seoul National University Bundang Hospital, Seongnam, ${ }^{\dagger}$ Department of Pediatrics, Seoul \\ National University College of Medicine, Seoul, Korea
}

Purpose: Clostridium difficile colonization and infection are commonly associated with poor outcomes in patients with pediatric inflammatory bowel disease (PIBD). We aimed to investigate the prevalence of $C$. difficile colonization and infection at the time of diagnosis and to evaluate risk factors associated with the development of $C$. difficile infection during the course of PIBD treatment.

Methods: We retrospectively enrolled a total of 59 children who were newly diagnosed with PIBD at the tertiary medical center. All patients underwent $C$. difficile toxin assays and cultures initially and at every follow-up during the disease course. Kaplan-Meier survival analysis and Cox regression test were used for statistical analysis.

Results: Initial cultures for $C$. difficile were positive in $13(22.0 \%)$ of 59 PIBD patients, whereas initial toxin assays were positive in 3 patients (5.1\%). During treatment, $C$. difficile cultures converted to positive in $28(47.5 \%)$ in addition to 13 patients who were initially culture-positive, and $C$. difficile toxins converted to positive in $13(22.0 \%)$ in addition to 3 originally toxin-positive patients. Antibiotic usage alone was significantly associated with the development of C. difficile colonization $(p=0.011)$, and the length of hospitalization was associated with the development of $C$. difficile infection $(p=0.032)$.

Conclusion: $C$. difficile colonization and infection occur frequently during the disease course of PIBD. Antibiotic usage and longer hospital stay were significant risks factors for the conversion of $C$. difficile status in PIBD patients undergoing treatment.

Key Words: Clostridium difficile, Infection, Inflammatory bowel diseases, Child

\section{INTRODUCTION}

Clostridium difficile is an anaerobic, gram-positive spore-forming bacillus causing a wide spectrum of diseases. Symptoms of $C$. difficile infection can range from mild, self-limited diarrhea to severe colitis with cramping, hematochezia, pseudomembrane formation, and intestinal perforation [1]. C. difficile col-

Received : October 9, 2017, Revised : December 14, 2017, Accepted : December 22, 2017

Corresponding author: Hye Ran Yang, Department of Pediatrics, Seoul National University Bundang Hospital, 82 Gumi-ro 173 beon-gil, Bundang-gu, Seongnam 13620, Korea. Tel: +82-31-787-7285, Fax: +82-31-787-4054, E-mail: hryang@snubh.org

Copyright (c) 2018 by The Korean Society of Pediatric Gastroenterology, Hepatology and Nutrition

This is an open-access article distributed under the terms of the Creative Commons Attribution Non-Commercial License (http://creativecommons.org/licenses/by-nc/4.0/) which permits unrestricted non-commercial use, distribution, and reproduction in any medium, provided the original work is properly cited. 
onizes the colon although the colonization itself is not invasive. Exotoxins (toxin A and toxin B) produced by the $C$. difficile bacillus mediate tissue injury and inflammation and cause symptoms associated with C. difficile infection [1].

Imbalances or alterations in gut microbiota composition (dysbiosis) have been reported in patients presenting with inflammatory bowel disease (IBD) [2], and IBD is associated with an increased prevalence of $C$. difficile infection [3]. Reportedly, the prevalence of $C$. difficile infection in pediatric patients diagnosed with IBD ranges between $3.5 \%$ and $69 \%$ [2,4-10].

C. difficile colonization and infection are common complications of IBD and are associated with poor outcomes [4,5]. Toxin producing $C$. difficile infection can trigger or worsen an IBD flare-up. Early diagnosis of $C$. difficile infection in IBD patients is thus very important. However, it is particularly difficult because clinical symptoms of $C$. difficile infection are indistinguishable from those of IBD flare-up [11]. Most of our current knowledge reading C. difficile colonization and infection in pediatric IBD (PIBD) patients is limited because most data have been obtained from adult studies and are associated with number of confounding factors $[4,12,13]$. Despite its clinical significance in PIBD, to date, C. difficile infection in PIBD has rarely been studied.

Therefore, the aims of our study were to investigate the prevalence of $C$. difficile colonization and infection in PIBD patients at the time of initial diagnosis and to evaluate the risk factors associated with C. difficile colonization and infection in PIBD patients during the disease course and treatment.

\section{MATERIALS AND METHODS}

\section{Subjects}

We enrolled a total of 59 children and adolescents who were newly diagnosed PIBD at the Seoul National University Bundang Hospital between March 2011 and May 2017. These patients were selected from the pediatric gastrointestinal disease database of our tertiary medical center. All enrolled patients underwent
C. difficile testing at the time of initial diagnosis and at every follow-up throughout the disease course. Demographic and clinical data were reviewed retrospectively in each patient.

This study was approved by the Institutional Review Board (IRB) of Seoul National University Bundang Hospital (IRB no. B-1603-338-101).

\section{Diagnosis of inflammatory bowel disease}

Diagnosis of Crohn disease (CD) and ulcerative colitis (UC) was based on clinical symptoms and signs, endoscopic and histopathological findings, as well as radiological investigations including magnetic resonance enterography (MRE). All enrolled patients underwent complete diagnostic work-up including colonoscopic examination with ileal intubation, upper gastrointestinal endoscopy, and MRE to evaluation of small intestine. Multiple biopsies were obtained in eachpatient from each segment of the gastrointestinal tract for histological evaluation.

\section{Diagnosis of Clostridium difficile infection}

All enrolled patients underwent $C$. difficile toxin assay testing and cultures. Toxin assay testing was performed using a stool enzyme immunoassay (EIA) kit (VIDAS ${ }^{\circledast}$ C. difficile Toxin A $\&$ B assay; BioMérieux, Marcy-l'Etoile, France). Cultures were performed using a specific stool culture medium $\left(\right.$ ChromID ${ }^{\circledR} \mathrm{C}$. difficile; BioMérieux).

C. difficile colonization was defined as a positive stool culture for $C$. difficile with a negative toxin immunoassay. C. difficile infection was defined as a condition wherein both the stool culture and toxin immunoassay for $C$. difficile were positive.

\section{Statistical analysis}

Data are presented as mean \pm standard deviation (SD) for parametric continuous variables and median (range) for nonparametric continuous variables. All data were analyzed using the IBM SPSS Statistics ver. 22.0 software program (IBM Co., Armonk, NY, USA).

The chi-squared test was used for statistical analysis of categorical variables compared between the 
groups. The Mann-Whitney U test for nonparametric analysis was used for analysis of continuous variables between the groups.

Kaplan-Meier survival analysis was performed to analyze the status of $C$. difficile during the disease course of PIBD. C. difficile colonization was defined as the event during the disease course on Kaplan-Meier survival analysis. Positive conversion of $C$. difficile culture and $C$. difficile toxin were set as a primary outcome of the Kaplan-Meier survival analysis in the present study. Kaplan-Meier survival curves were generated and statistical significance was analyzed.

Cox regression tests were used to analyze of risk factors associated with $C$. difficile colonization or infection during the treatment course of PIBD. Patients who initially tested positive for C. difficile toxin or culture were excluded from the Cox regression analysis. Using the R program, the Cox hazard model was created by plotting a graph of the relative log of the hazard ratio. All $p$-values $<0.05$ were considered statisti-

Table 1. Baseline Clinical Features of the Patients Recruited

\begin{tabular}{lc}
\hline \multicolumn{1}{c}{ Variable } & IBD $(\mathrm{n}=59)$ \\
\hline Male gender & $38(64.4)$ \\
Age $(\mathrm{y})$ & $13.5 \pm 2.6$ \\
Initial $C$. difficile culture positivity & $13(22.0)$ \\
Initial C. difficile toxin positivity & $3(5.1)$ \\
\hline
\end{tabular}

Values are presented as number (\%) or mean \pm standard deviation. IBD: inflammatory bowel disease, $C$. difficile: Clostridium difficile. cally significant.

\section{RESULTS}

\section{Patient characteristics}

Our study included 59 children and adolescents ( 38 boys, 21 girls; mean age $\pm S D, 13.5 \pm 2.6$ years) who were newly diagnosed with IBD and underwent C. difficile testing at their first visit. Demographic and clinical data of the PIBD patients recruited are listed in Table 1.

\section{The status of $C$. difficile infection of patients with pediatric inflammatory bowel disease at the time of diagnosis and during the disease course}

C. difficile cultures were positive at the time ofinitial diagnosis in 13 patients $(22.0 \%)$ of 59 children with PIBD, whereas the $C$. difficile toxin assay at the time of diagnosis was positive in only 3 patients (5.1\%) of 59 PIBD patients (Table 1 ).

Thirteen patients $(22.0 \%)$ were initially culturepositive at the time of diagnosis, and an additional 28 patients $(47.5 \%)$ showed conversion to positive cultures during the disease course of IBD. Thus, C. difficile cultures were positive in a total of $41(69.5 \%)$ patients with PIBD.

Only 3 patients (5.1\%) were initially toxin-positive at the time of diagnosis, and toxins assay tests converted to positive result in an additional 13 patients

Table 2. Comparison of Clinical Factors between Children with CD and Those with UC

\begin{tabular}{lccc}
\hline \multicolumn{1}{c}{ Variable } & CD $(\mathrm{n}=41)$ & UC $(\mathrm{n}=18)$ & $p$-value \\
\hline Male gender & $27(65.9)$ & $11(61.1)$ & 0.726 \\
Age $(\mathrm{y})$ & $13.5 \pm 2.5$ & $13.4 \pm 2.8$ & 0.833 \\
Initial C. difficile culture positivity & $10(24.4)$ & $3(16.7)$ & 0.735 \\
Initial C. difficile toxin positivity & $2(4.9)$ & $1(5.6)$ & 1.000 \\
Colon involvement & $29(70.7)$ & $18(100.0)$ & 0.012 \\
No. of patients who used antibiotics during the disease course & $5(12.2)$ & $1(5.6)$ & 0.656 \\
No. of patients who underwent operation during the disease course & $4(9.8)$ & $0(0)$ & 0.303 \\
The total duration of admission (d) & $38.1 \pm 41.0$ & $15.3 \pm 18.0$ & 0.025 \\
Frequency of disease flare during the disease course & $1.3 \pm 0.8$ & $1.1 \pm 1.0$ & 0.459 \\
\hline
\end{tabular}

Values are presented as number (\%) or mean \pm standard deviation.

CD: Crohn disease, UC: ulcerative colitis, C. difficile: Clostridium difficile. $p$-value less than 0.05 was set to be statistically significant. 
(22.0\%) during the course of PIBD. Thus, C. difficile toxin assays were positive in a total of $16(27.1 \%)$ patients with IBD.

Clinical features and the status of $C$. difficile infection in pediatric patients diagnosed with Crohn disease and ulcerative colitis at the time of diagnosis and during disease course

Among the 59 newly diagnosed PIBD patients, 41 patients $(69.5 \%)$ had CD and 18 patients $(30.5 \%)$ had UC. Factors that can affect the status of $C$. difficile infection in patients diagnosed with IBD such as colonic involvement, antibiotics use, history of operations, length of hospitalization, and frequency of disease flare-ups were listed and compared between the patients with CD and those with UC (Table 2). Only the length of hospitalization revealed a statistically significant difference between the groups (Table 2).

Kaplan-Meier survival analysis was performed in patients with $\mathrm{CD}$ and $\mathrm{UC}$, respectively, for $C$. difficile colonization during IBD treatment, which revealed no statistically significant difference between those with CD and UC $(p=0.332)$ although C. difficile colonization with culture positivity developed in both groups during the disease course of IBD (Fig. 1).

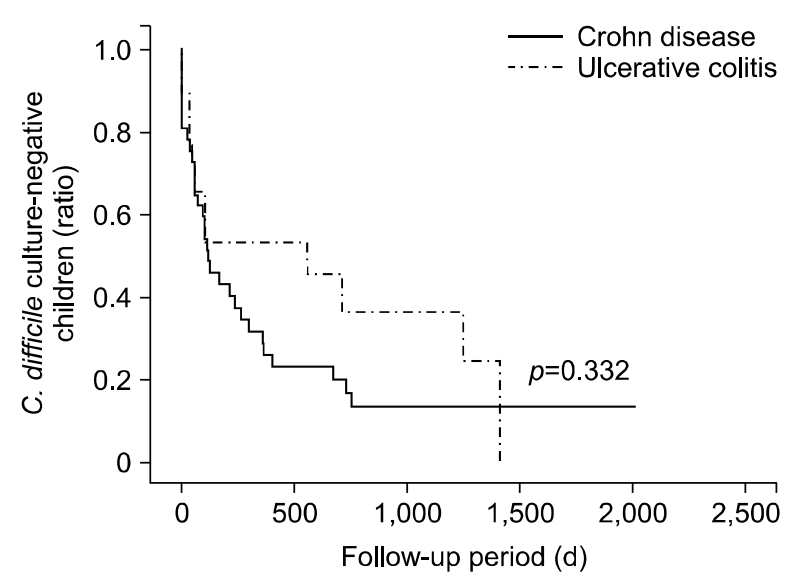

Fig. 1. Survival curve for Clostridium difficile colonization with culture positivity during the disease course of inflammatory bowel disease in children with Crohn disease and those with ulcerative colitis, respectively. As C. difficile colonization was defined as the event for survival analysis, y axis of the graph indicates the ratio of $C$. difficile culture-negative children.
In the same way, Kaplan-Meier survival analysis was also performed in patients with $\mathrm{CD}$ and $\mathrm{UC}$ for $C$. difficile infection during the disease course of IBD, which revealed no statistically significant difference between those with CD and UC ( $p=0.672)$ although C. difficile infection with both toxin and culture positivity developed in both groups during the disease course (Fig. 2).

Factors affecting the development of $C$. difficile colonization and infection during the treatment of pediatric inflammatory bowel disease

Based on the results obtained from univariate analysis, Cox regression analysis was performed using significant variables such as the type of IBD, colonic involvement, antibiotic use, history of operations, length of hospitalization, and the frequency of disease flare-ups as possible risk factors associated with the development of $C$. difficile colonization and infection. Use of antibiotics was the sole significant risk factor associated with fecal conversion to $C$. difficile colonization ( $p=0.011)$ (Table 3$)$. Length of hospitalization was the sole risk factor associated with fecal conversion to $C$. difficile infectionin PIBD pa-

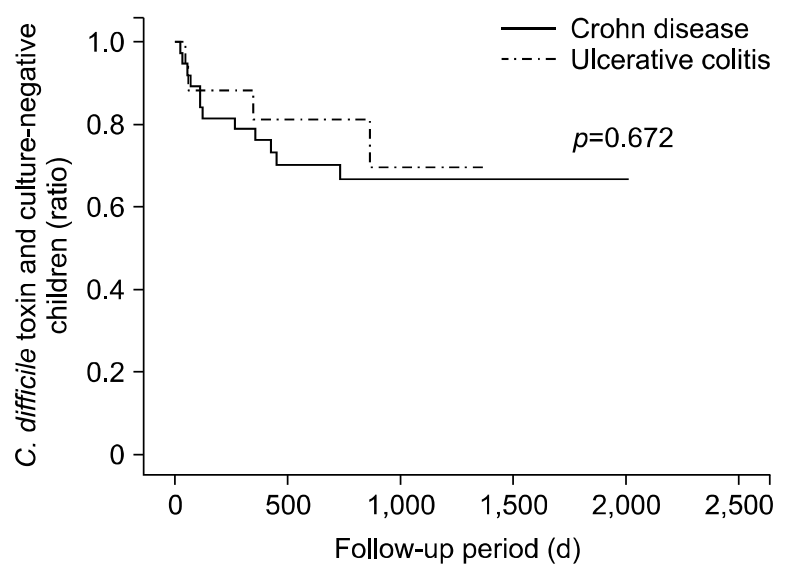

Fig. 2. Survival curve for Clostridium difficile infection with toxin and culture positivity during the disease course of inflammatory bowel disease in children with Crohn disease and those with ulcerative colitis, respectively. As $C$. difficile infection was defined as the event for survival analysis, y axis of the graph indicates the ratio of $C$. difficile toxin and culturenegative children. 
Table 3. Risk Factors for Developing Clostridium difficile Colonization and C. difficile Infection during the Disease Course of Pediatric Inflammatory Bowel Disease

\begin{tabular}{llccc}
\hline & \multicolumn{1}{c}{ Variable } & Exp(B) & 95\% confidence interval & $p$-value \\
\hline C. difficile colonization & Antibiotic usage & 3.754 & $1.360-10.366$ & 0.011 \\
C. difficile infection & The duration of admission & 1.018 & $1.002-1.035$ & 0.032 \\
\hline
\end{tabular}

$p$-value less than 0.05 was set to be statistically significant.

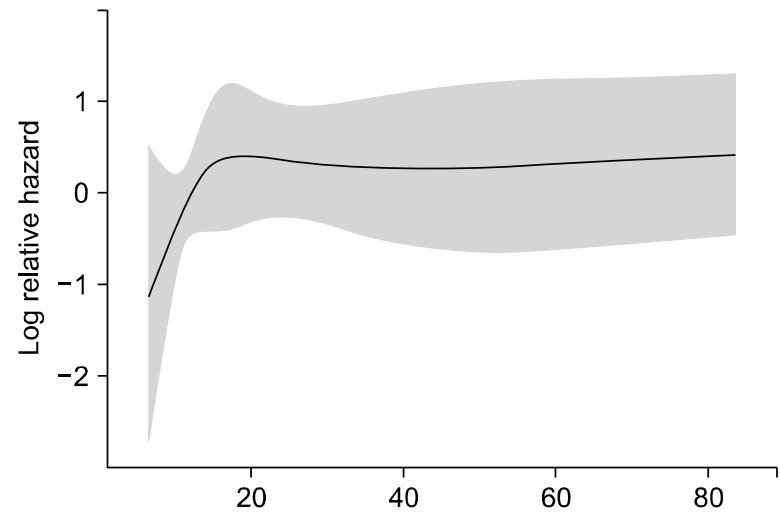

(d)

Fig. 3. Relative hazard ratio graph (log scale) of Clostridium difficile infection according to total hospitalization period in children with inflammatory bowel disease.

tients $(p=0.032)$ (Table 3, Fig. 3).

\section{DISCUSSION}

In the present study, we evaluated the status of $C$. difficile infection at the time of initial diagnosis of PIBD and investigated the risk factors associated with $C$. difficile colonization and infection during the disease course and IBD treatment. Our results revealed that initial culture positivity at the time of diagnosis was observed in approximately $22 \%$ of patients with PIBD, whereas C. difficile toxin positivity at the time of initial diagnosis was noted only in approximately $5 \%$ of patients with PIBD. Furthermore, C. difficile cultures converted to positive results in approximately $47.5 \%$ of PIBD patients during the disease course of PIBD in those receiving treatment. Thus, approximately $70 \%$ of PIBD patients eventually demonstrated $C$. difficile culture positivity at least once. Additionally, C. difficile toxin assays also con- verted to show positive results in $22.0 \%$ of the patients during the disease course of PIBD. Thus, approximately $27.1 \%$ of PIBD patients finally demonstrated toxin positivity at least once. According to a previous study, C. difficile colonization and infection were observed to be more prevalent in patients with PIBD than children without PIBD, and subsequent conversion to $C$. difficile colonization and infection occurred frequently during PIBD treatment even in those with a negative $C$. difficile status at the time of diagnosis [14].

Recent studies reported a significantly increased prevalence of $C$. difficile infection in patients with IBD [9,15-17], particularly in the pediatric age group, ranging between 16 and $69 \%[2,6,18,19]$. The high prevalence of $C$. difficile colonization and infection in IBD patients could be attributed to the fact that dysbiosis is a risk factor for the development of both, IBD and $C$. difficile infection $[20,21]$. Our study showed a positive $C$. difficile colonization rate of $22.0 \%$ and a $C$. difficile infection rate of $5.1 \%$ with both culture and toxin positivity at the time of initial diagnosis and total rates (infection rate at the time of diagnosis combined with the conversion rate during the disease course) were $69.5 \%$ and $27.1 \%$, respectively. This significant discrepancy observed between related studies could be because of the extremely low sensitivity of thetoxin immunoassay used to detect $C$. difficile infection and other unidentified local factors $[22,23]$. Another study revealed that the sensitivity of the $C$. difficile toxin assay in immunocompromised patients was very low, and this can be similarly applied to IBD patients treated with methotrexate and thiopurines [24].

Previous studies have demonstrated that IBD itself is an independent risk factor for $C$. difficile in- 
fection in both adult and pediatric populations $[2,11,12]$. In this study, the prevalence of $C$. difficile colonization was significantly higher in patients with PIBD than other children at the time of initial diagnosis [14]. Additionally, C. difficile colonization and infection were not related to the type of IBD in our study - this finding was consistent with the results reported by other previous studies $[8,9]$.

Because IBD is a chronic gastrointestinal disease, its treatment continues without interruption as the disease runs a life-long course. Thus, the conversion of $C$. difficile status during IBD treatment can be a critical issue in PIBD patient. Based on previous studies, the status of $C$. difficile infection is routinely monitored for 1-2 years or at specific time-points such as time IBD is first diagnosed or at the time of an IBD flare-up $[9,23,25]$. However, no studies have reported the conversion of $C$. difficile status during the disease course of IBD yet. Therefore, our study followed up PIBD patients for a relatively long period of time. Thus, we could identify the conversion rate and the pattern of $C$. difficile infection. Despite the limitations secondary to the retrospective nature of our study, C. difficile colonization, which might have affected disease progression in PIBD patients, was observed in most patients over a 6-year observation period.

Although various risk factors associated with $C$. difficile infection have been reported, to date, it is unclear which factors other than IBD itself affect the development of $C$. difficile infection in IBD patients. Our present study showed that among, several factors studied, antibiotic use was the only significant risk factor associated with the occurrence of $C$. difficile colonization during the course of PIBD in patients receiving treatment, and that the length of hospitalization was a significant risk factor associated with $C$. difficile infection during PIBD treatment. Longer the period of hospitalization and higher the severity of the disease, greater was the risk of $C$. difficile infection among patients in a hospital setting. Moreover, we observed that $C$. difficile infection in patients with IBD affects the clinical course of IBD and increases the length of hospitalization [26]. Therefore, it is important in clinical practice to adopt systematized strategies to prevent the vicious cycle of recurrent $C$. difficile infection superimposed on the disease course in PIBD patients.

Usually, antibiotic use is considered as the primary risk factor associated with $C$. difficile infection. Previous studies have not reported a statistically significant effect of antibiotic usage on the development of $C$. difficile infection in IBD patients as was shown by our study $[2,6,27]$. However, our present study demonstrated that the use of antibiotics was clearly associated with $C$. difficile colonization during the disease course of PIBD. C. difficile infection occurs as a result of $C$. difficile colonization; therefore, antibiotic use should be avoided or minimized in patients with IBD to prevent $C$. difficile colonization and subsequent infection.

Limitations of our study: 1) ours was a single center study with a retrospective design. The number of patients recruited in this study was relatively small; thus, a few factors that are known to be risks factors for development of $C$. difficile infection did not show statistically significant result in this study. Further studies involving a larger number of patients are needed to conclusively establish results in this context. 2) The sensitivity of the toxin assay performed using the EIA method for the diagnosis of $C$. difficile infection is very low $[19,23,24]$. Therefore, a few patients with $C$. difficile infection might have been incorrectly classified as those with $C$. difficile colonization rather than an infection. Although a polymerase chain reaction test is a relatively accurate to detect $C$. difficile, it is an expensive and time-consuming method for routine use in clinical practice [28].

In conclusion, $C$. difficile colonization is prevalent at the time of initial diagnosis of PIBD, and C. difficile colonization and infection can occur frequently during the disease course of PIBD. Antibiotic usage and longer hospitalization were observed to be significant risks factors associated with for the conversion of $C$. difficile status in PIBD patients during the disease course. Therefore, early and accurate diagnosis of $C$. difficile infection in PIBD patients based on the risk factors suggested in the present study 
may facilitate prompt and correct diagnosis and institution of appropriate treatment strategies in PIBD patients and thereby improve the prognosis of PIBD.

\section{ACKNOWLEDGEMENTS}

There are no conflicts of interest to declare. The authors thank Dr. JB Lee and the Division of Statistics at the Medical Research Collaborating Center, Seoul National University Bundang Hospital for the assistance with statistical analysis.

\section{REFERENCES}

1. Kelly CP, Pothoulakis C, LaMont JT. Clostridium difficile colitis. N Engl J Med 1994;330:257-62.

2. Pascarella F, Martinelli M, Miele E, Del Pezzo M, Roscetto E, Staiano A. Impact of Clostridium difficile infection on pediatric inflammatory bowel disease. J Pediatr 2009;154:854-8.

3. Sokol H, Lay C, Seksik P, Tannock GW. Analysis of bacterial bowel communities of IBD patients: what has it revealed? Inflamm Bowel Dis 2008;14:858-67.

4. Ananthakrishnan AN, McGinley EL, Saeian K, Binion DG. Temporal trends in disease outcomes related to Clostridium difficile infection in patients with inflammatory bowel disease. Inflamm Bowel Dis 2011; 17:976-83.

5. Mezoff E, Mann EA, Hart KW, Lindsell CJ, Cohen MB. Clostridium difficile infection and treatment in the pediatric inflammatory bowel disease population. $\mathrm{J}$ Pediatr Gastroenterol Nutr 2011;52:437-41.

6. Wultańska D, Banaszkiewicz A, Radzikowski A, ObuchWoszczatyński P, Młynarczyk G, Brazier JS, et al. Clostridium difficile infection in Polish pediatric outpatients with inflammatory bowel disease. Eur J Clin Microbiol Infect Dis 2010;29:1265-70.

7. Kelsen JR, Kim J, Latta D, Smathers S, McGowan KL, Zaoutis T, et al. Recurrence rate of Clostridium difficile infection in hospitalized pediatric patients with inflammatory bowel disease. Inflamm Bowel Dis 2011; 17:50-5.

8. Pant C, Anderson MP, Deshpande A, Altaf MA, Grunow JE, Atreja A, et al. Health care burden of Clostridium difficile infection in hospitalized children with inflammatory bowel disease. Inflamm Bowel Dis 2013; 19:1080-5.

9. Banaszkiewicz A, Kowalska-Duplaga K, Pytrus T, Pituch H, Radzikowski A. Clostridium difficile in- fection in newly diagnosed pediatric patients with inflammatory bowel disease: prevalence and risk factors. Inflamm Bowel Dis 2012;18:844-8.

10. Banaszkiewicz A, Pituch H. Clostridium difficile infection in children with inflammatory bowel disease: current evidence. Curr Pharm Des 2014;20:4549-55.

11. Issa M, Vijayapal A, Graham MB, Beaulieu DB, Otterson MF, Lundeen S, et al. Impact of Clostridium difficile on inflammatory bowel disease. Clin Gastroenterol Hepatol 2007;5:345-51.

12. Rodemann JF, Dubberke ER, Reske KA, Seo DH, Stone CD. Incidence of Clostridium difficile infection in inflammatory bowel disease. Clin Gastroenterol Hepatol 2007;5:339-44.

13. Nguyen GC, Kaplan GG, Harris ML, Brant SR. A national survey of the prevalence and impact of Clostridium difficile infection among hospitalized inflammatory bowel disease patients. Am J Gastroenterol 2008;103:1443-50.

14. Schutze GE, Willoughby RE; Committee on Infectious Diseases; American Academy of Pediatrics. Clostridium difficile infection in infants and children. Pediatrics 2013;131:196-200.

15. Ananthakrishnan AN, Issa M, Binion DG. Clostridium difficile and inflammatory bowel disease. Gastroenterol Clin North Am 2009;38:711-28.

16. Musa S, Thomson S, Cowan M, Rahman T. Clostridium difficile infection and inflammatory bowel disease. Scand J Gastroenterol 2010;45:261-72.

17. Goodhand JR, Alazawi W, Rampton DS. Systematic review: Clostridium difficile and inflammatory bowel disease. Aliment Pharmacol Ther 2011;33:428-41.

18. Gryboski JD. Clostridium difficile in inflammatory bowel disease relapse. J Pediatr Gastroenterol Nutr 1991;13:39-41.

19. Markowitz JE, Brown KA, Mamula P, Drott HR, Piccoli DA, Baldassano RN. Failure of single-toxin assays to detect Clostridium difficile infection in pediatric inflammatory bowel disease. Am J Gastroenterol 2001; 96:2688-90.

20. Monaghan TM, Cockayne A, Mahida YR. Pathogenesis of Clostridium difficile infection and its potential role in inflammatory bowel disease. Inflamm Bowel Dis 2015;21:1957-66.

21. Gómez S, Chaves F, Orellana MA. Clinical, epidemiological and microbiological characteristics of relapse and re-infection in Clostridium difficile infection. Anaerobe 2017;48:147-51.

22. Ott C, Girlich C, Klebl F, Plentz A, Iesalnieks I, Schölmerich J, et al. Low risk of Clostridium difficile infections in hospitalized patients with inflammatory 
bowel disease in a German tertiary referral center. Digestion 2011;84:187-92.

23. Sokol H, Lalande V, Landman C, Bourrier A, Nion-Larmurier I, Rajca S, et al. Clostridium difficile infection in acute flares of inflammatory bowel disease: a prospective study. Dig Liver Dis 2017;49:643-6.

24. Erb S, Frei R, Strandén AM, Dangel M, TschudinSutter S, Widmer AF. Low sensitivity of fecal toxin A/B enzyme immunoassay for diagnosis of Clostridium difficile infection in immunocompromised patients. Clin Microbiol Infect 2015;21:998.e9-15.

25. Martinelli M, Strisciuglio C, Veres G, Paerregaard A, Pavic AM, Aloi M, et al. Clostridium difficile and pediatric inflammatory bowel disease: a prospective, comparative, multicenter, ESPGHAN study. Inflamm Bowel Dis 2014;20:2219-25.
26. Saffouri G, Gupta A, Loftus EV Jr, Baddour LM, Pardi DS, Khanna S. The incidence and outcomes from Clostridium difficile infection in hospitalized adults with inflammatory bowel disease. Scand J Gastroenterol 2017;52:1240-7.

27. Hojsak I, Ferenc T, Bojanić K, Mišak Z, Močić Pavić A, et al. Incidence of Clostridium difficile infection in children with inflammatory bowel disease compared to oncology and immunocompetent patients. Digestion 2012; 86:6-11.

28. Peterson LR, Manson RU, Paule SM, Hacek DM, Robicsek A, Thomson RB Jr, et al. Detection of toxigenic Clostridium difficile in stool samples by real-time polymerase chain reaction for the diagnosis of C. difficile-associated diarrhea. Clin Infect Dis 2007;45:1152-60. 